\title{
Herausforderung Sucht
}

\author{
In den Achtzigerjahren erlangte die Schweiz durch ihre offenen Drogenszenen \\ traurige Berühmtheit. Sie reagierte mit revolutionären Massnahmen gegen das \\ Elend der Süchtigen. Wo steht die Suchtmedizin heute?
}

Erika Jüsi

Korrespondenz:

Erika Jüsi

für Suchtmedizin

Konradstrasse 32

CH-8005 Zürich

e.juesi[at]arud.ch

www.arud.ch
Arud Zentren

Tel. 0583605050

Fax 0583605019

Eine Institution, die sich schon seit zwanzig Jahren intensiv mit den Herausforderungen in der Suchtmedizin beschäftigt, ist die Arud: Eine Handvoll Ärzte gründet am 30. November 1991 eine Arbeitsgemeinschaft, um mit einem niederschwelligen Methadonangebot möglichst vielen Süchtigen eine Substitutionstherapie zu ermöglichen. Mit der Stadt Zürich im Rücken eröffnet die Arud im Februar 1992 - unmittelbar nach der Platzspitzschliessung - das Zokl1 (heute Zentrum Aussersihl) und wird von Behandlungswilligen überrannt. Nach nur drei Monaten sind bereits 300 Substitutionspatienten angemeldet, nach einem Jahr beziehen 500 im Zokl1 regelmässig ihr Methadon. Zwei Jahre später geht die Arud einen Schritt weiter und eröffnet das Zokl2 (heute Zentrum Stampfenbach), wo sie mit dem weltweit ersten Programm für heroingestützte Behandlungen beginnt. Die Stadt Zürich zieht nur wenige Wochen später mit einem eigenen Angebot nach.

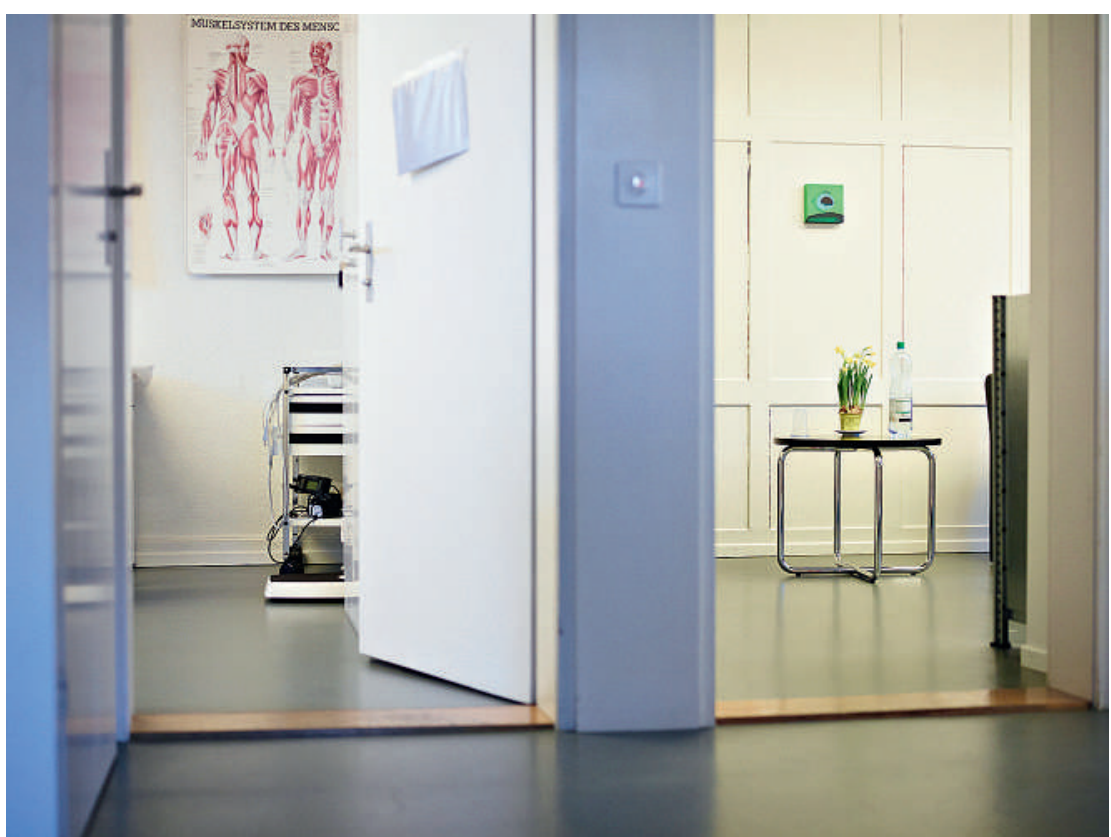

Erfahrene und auf Substanzabhängigkeiten spezialisierte Teams bieten in den Zentren der Arud ein interdisziplinäres Angebot unter einem Dach.

\section{Der enorme Druck ermöglichte progressive Lösungen}

Die Städte Zürich, Bern und Basel, unter enormem Druck durch ihre offenen Drogenszenen mitten in den Stadtzentren, waren massgeblich an den Innovationen beteiligt, die zu einem weltweit beachteten, komplett neuen Ansatz im Umgang mit dem in ganz Europa verbreiteten Drogenproblem führten und Substitutionstherapien ermöglichten. Auch der Bund musste in Anbetracht des Elends, das die offenen Drogenszenen in den Städten offenbarten, die Notwendigkeit und absolute Dringlichkeit eines neuen Lösungsansatzes einsehen. Alle bereits versuchten Massnahmen hatten nicht die erhoffte Entspannung gebracht. Während damals die traurigen Bilder des Zürcher «Needle Parks», der grössten offenen Drogenszene in der Schweiz und Europa, die Welt schockierten, hospitieren heute Delegationen aus der ganzen Welt in den Schweizer Zentren für Suchtmedizin, um von ihnen zu lernen.

\section{Heroin kein Thema mehr}

Heroin ist als Substanz in den letzten Jahren stark in den Hintergrund getreten. Die Substitutionspatienten sind älter geworden. Dr. Thilo Beck, Chefarzt Psychiatrie der Arud: «Diejenigen, die den Absprung bis heute nicht geschafft haben, leiden oft unter schweren psychischen Störungen oder Traumata, die bis in die frühe Kindheit zurückgehen.» Neue Heroinabhängige gebe es eher selten. Auch bei der Arud stehen heute Substanzen wie Alkohol, Kokain, Medikamente, Nikotin oder Cannabis im Vordergrund.

\section{Das Ziel der integrierten Versorgung}

Suchtpatienten werden heute entweder von Hausärzten oder in diversen spezialisierten Zentren und Einrichtungen betreut. Der Vorteil einer Behandlung in den Zentren ist, dass ein interdisziplinäres, auf Substanzabhängigkeiten spezialisiertes Team von Internisten/Allgemeinmedizinern, Psychiatern und Sozialarbeitern den oft vielschichtigen Problemen von Suchtpatienten gerecht werden kann. 


\section{Zur Arud}

1991 gegründet als «Arbeitsgemeinschaft für den risikoarmen Umgang mit Drogen», heute «Zentren für Suchtmedizin». Sie bietet ein interdisziplinäres Angebot über alle Substanzen hinweg für Konsumierende, Ärzte und Fachleute, Angehörige und Unternehmen. Sie ist Mitverfasserin von nationalen Guidelines im Substitutions- und Hepatitis-C-Bereich; in Letzterem ist sie federführend im Engagement für eine nationale und internationale Verbesserung der medizinisch-infektiologischen Versorgung von Suchtpatienten.

Hausärzte sind für viele Patienten mit Abhängigkeiten wichtige Bezugspersonen und unentbehrlich im Versorgungsnetz der Suchtmedizin. Im Idealfall arbeiten sie mit den Zentren zusammen, holen sich

\section{«Hausärzte sind für viele Patienten mit Abhängig- keiten wichtige Bezugspersonen und unentbehrlich im Versorgungsnetz der Suchtmedizin.»}

wenn nötig Unterstützung oder überweisen Patienten, wenn die Infrastruktur der eigenen Praxis nicht mehr ausreicht. Diese Zusammenarbeit gilt es laut Dr. Beck noch zu intensivieren. «Wir müssen versuchen, die Suchtmedizin besser in das bestehende Versorgungsnetz zu integrieren. Das ist im Moment noch nicht zufriedenstellend umgesetzt.» Die Arud bietet fachliche Unterstützung und Beratung an und unterstützt aktiv den Aufbau entsprechender Netzwerke.
Thilo Beck sieht auch bei der Art der Behandlungen im Suchtbereich Verbesserungspotential. Die Therapien müssten besser auf die einzelnen Patienten abgestimmt werden, sagt er. «Man kann nicht einfach ein Programm fahren, das dann für alle angewendet wird.» Die Interdisziplinarität spiele da eine wichtige Rolle. Bei einer engen Zusammenarbeit einzelner Fachbereiche und Berufsgruppen muss allerdings der Dialog zwischen den einzelnen Stellen funktionieren, damit sie voneinander profitieren. «Hier gilt es, die Schnittstellenproblematik in den Griff zu bekommen.»

\section{Sucht als Stigma}

Eine weitere Herausforderung liegt gemäss Thilo Beck in der kontinuierlichen Aufrechterhaltung und Weiterentwicklung eines flächendeckenden niederschwelligen Angebots. «Bei Substanzstörungen soll möglichst schnell und unkompliziert eine auf die Bedürfnisse der Patienten abgestimmte Therapie möglich sein, und zwar in der ganzen Schweiz.» Erstens sind auch nach der Revision des Betäubungsmittelgesetzes die gesetzlichen Rahmenbedingungen einschränkend, und zweitens gibt es bis heute Kantone, die mit einer restriktiven Suchtpolitik den Aufbau einer adäquaten Versorgung verhindern. Dies habe mit der in vielen Gebieten noch heute vorherrschenden Diskriminierung der Suchtkranken zu tun. «Es muss endlich eine Entstigmatisierung von Substanzstörungen erreicht werden. Nur so kann eine kohärente Suchtpolitik entstehen.»

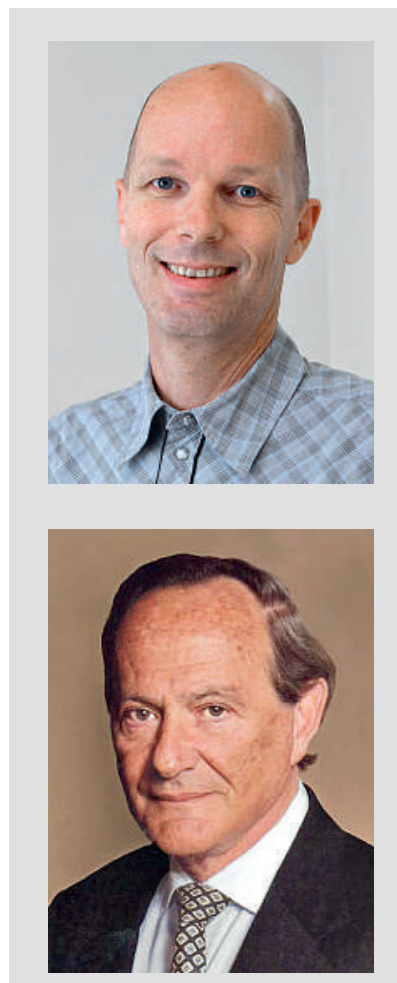

Dr. med. Thilo Beck, Chefarzt Psychiatrie der Arud seit 2007: «Wir müssen schweizweit eine flächendeckende niederschwellige Suchtversorgung anstreben.»
Professor Ambros Uchtenhagen,

Gründer des Instituts für Sucht- und Gesundheitsforschung ISGF und Präsident der Stiftung für Suchtforschung:

«Die Arud ist bis heute ein unentbehrlicher Motor für die Suchtmedizin in der Schweiz.»

Dr. med. André Seidenberg, Mitgründer der Arud «Unser Ziel war ein legaler Markt: Alle Substanzprobleme sollten in medizinische Hände, die Abgabe über Ärzte, Apotheken erfolgen.»

Professor Michael Krausz, MD, PhD, FRCPC, Medical Director Treatment Center for Mental Health and Addiction, Vancouver:

«Der Beitrag der Arud zur Innovation in einem dynamischen Bereich ist ein besonderes Beispiel gelungenen patientenorientierten Engagements.»

Dr. med. Felix Gutzwiller, Präventivmediziner und Politiker:

«Auch in der Zukunft wird die sich wandelnde Drogenproblematik immer wieder nach neuen Ansätzen in Prävention, Therapie und Rehabilitation verlangen. Arud ist für diese Rolle gut positioniert.» 